\title{
Glissonian Capsule Fistula after Laparoscopic Cholecystectomy
}

\author{
Assem Hajj,, Haydar A. Nasser, ${ }^{2}$ Ihab Joudi, Dany Chalhoub, ${ }^{3}$ and Claude Tayar ${ }^{1}$
}

\begin{abstract}
Background: Perforated gallbladder is a common complication during laparoscopic cholecystectomy. Although morbidity is rare, serious complications can manifest subsequently. Patients may suffer long-term complications and laparotomy has been suggested to some for symptom relief.

Patients and Methods: We present a case of laparoscopic cholecystectomy that was complicated by gallstone spillage. The patient presented later with a fistula, connecting the Glisson capsule to the subxiphoid trocar scar. Laparoscopic resection of the fistulous tract was recommended to the patient.

Results: The post-operative course was uneventful and the patient remained asymptomatic 12 months after the procedure.

Conclusion: Perforated gallbladder is a common complication during laparoscopic cholecystectomy and patients may suffer long-term complications. Some have had laparotomy recommended for symptom relief. However, recent research could not determine the best treatment modality. We have offered re-laparoscopy with concomitant excision of the fistulous tract that showed good results.
\end{abstract}

\section{Introduction}

B ILE DUCT INJURY and post-cholecystectomy syndrome are the most frequently studied complications regarding laparoscopic cholecystectomy morbidity. Although gallbladder perforation is encountered frequently during surgery [1], the consequences of such perforations have not been well evaluated. More than 100 articles are available on PubMed, most of which are case reports. According to Woodfield et al. [2], perforation could result in lost gallstones in approximately $7.3 \%$ of cases. These lost or spilled gallstones can lead to complications. In one prospective study, 49 patients suffered retained intra-abdominal stones [3]. Of these 49 patients, nine presented with complications that manifested later. Contrary to bile leakage, which can manifest soon after laparoscopic cholecystectomy, complications related to spilled gallstones present late after the procedure. Tumer et al. [3] reported seven complicated cases, in which symptoms occurred between one and $128 \mathrm{wks}$. We present a case of a complicated retained abdominal gallstone that resulted in cutaneous fistula formation three months after the procedure. Laparoscopic lavage combined with fistula tract excision was done.

\section{Case Report}

A 67-year-old female, who smoked and was hypertensive, underwent laparoscopic cholecystectomy for acute cholecystitis. During the operation a tear occurred at the level of the infundibulum. This was grasped and dissection continued appropriately. During exteriorization of the gallbladder through the subxiphoid trocar site, spillage of small gallstones occurred. Extraction of these spilled stones was done; this was followed by heavy irrigation and aspiration. The post-operative course was uneventful and the patient was discharged home on the second post-operative day. Three months later, the patient started to complain of bile draining in small quantity from the subxipoid scar. Computed tomography (CT) scan showed a well-demarcated fistulous tract between the skin and the liver capsule (Fig. 1). The fistulous tract was not communicating with the intra-hepatic biliary ducts.

Cultures were taken that grew no bacteria. Daily dressing changes for one week did not improve her condition. The decision was made to proceed with laparoscopic resection of the fistulous tract. This option was discussed with the patient and she signed the informed consent. During laparoscopy, the

\footnotetext{
${ }^{1}$ Department of General Surgery, Clemenceau Medical Center, Beirut, Lebanon.

${ }^{2}$ Department of General Surgery, Lebanese University, Beirut, Lebanon.

${ }^{3}$ Faculty of Medicine, Saint-Joseph University, Beirut, Lebanon.

(C) Assem Hajj et al. 2016; Published by Mary Ann Liebert, Inc. This Open Access article is distributed under the terms of the Creative Commons License (http://creativecommons.org/licenses/by/4.0), which permits unrestricted use, distribution, and reproduction in any medium, provided the original work is properly credited.
} 


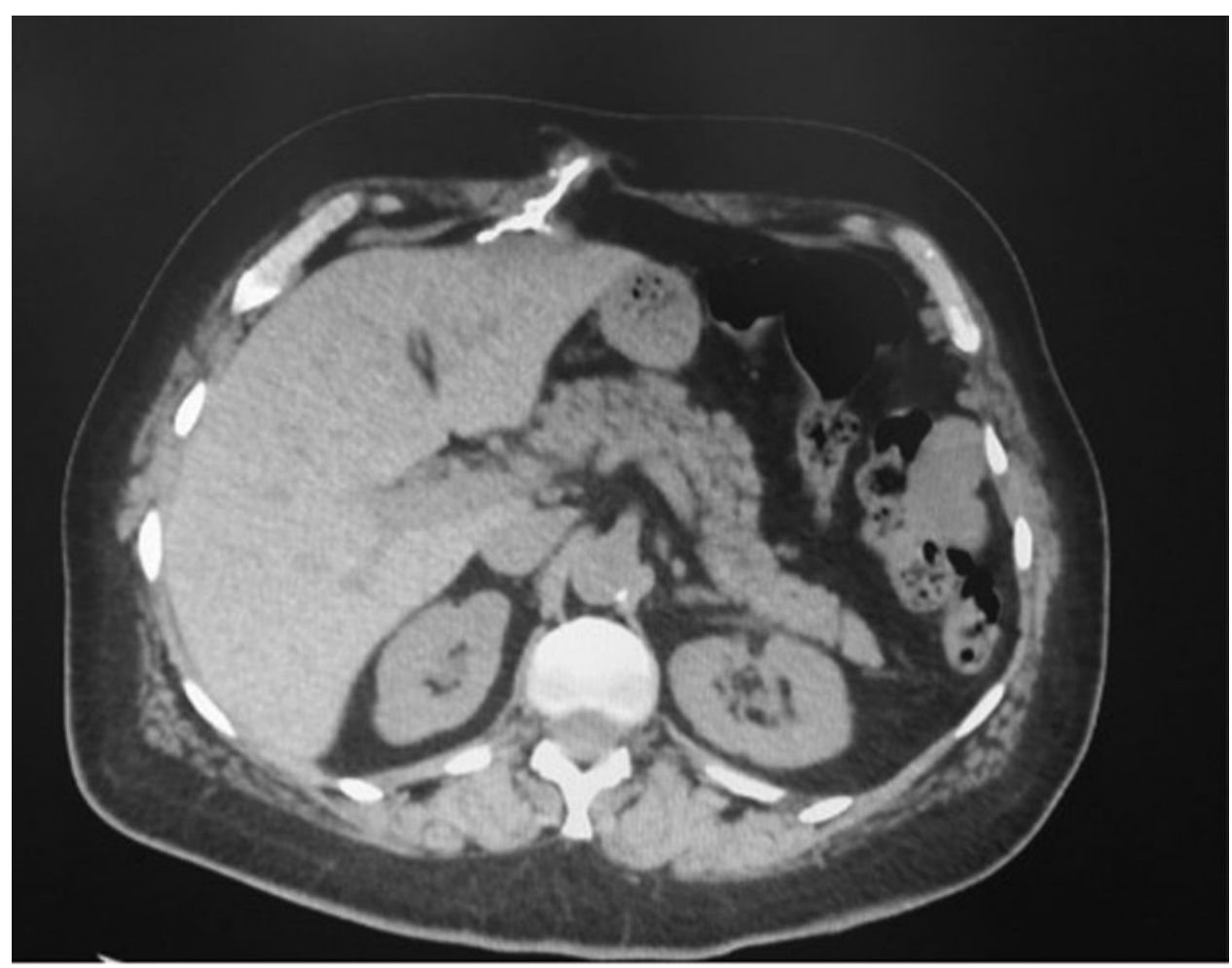

FIG. 1. Computed tomography scan of the abdomen showing the fistulous tract.

same previous incisions were used, except for the subxiphoid one in which no trocar had been introduced. The fistulous tract was visualized (Fig. 2) and its base at the Glisson capsule level was completely excised, using ultrasonic energy device (Fig. 3). An elliptical incision was then made around the external fistulous pit, which was deepened to the level of the parietal peritoneum. This elliptical cut with the fistulous tract attached to it were removed en bloc. At the conclusion of the procedure, a drain was positioned in the sub-hepatic space. It remained in place until less than $20 \mathrm{~mL}$ drained and was removed on the fourth post-operative day. There was no evidence of bile in the drain reservoir during that period. The post-operative course was uneventful and the patient remained symptom-free 12 months after the procedure.

\section{Discussion}

Perforated gallbladder during laparoscopic cholecystectomy is a common complication. Spilled gallstones can result in complications in approximately $2.3 \%$ of cases, however,

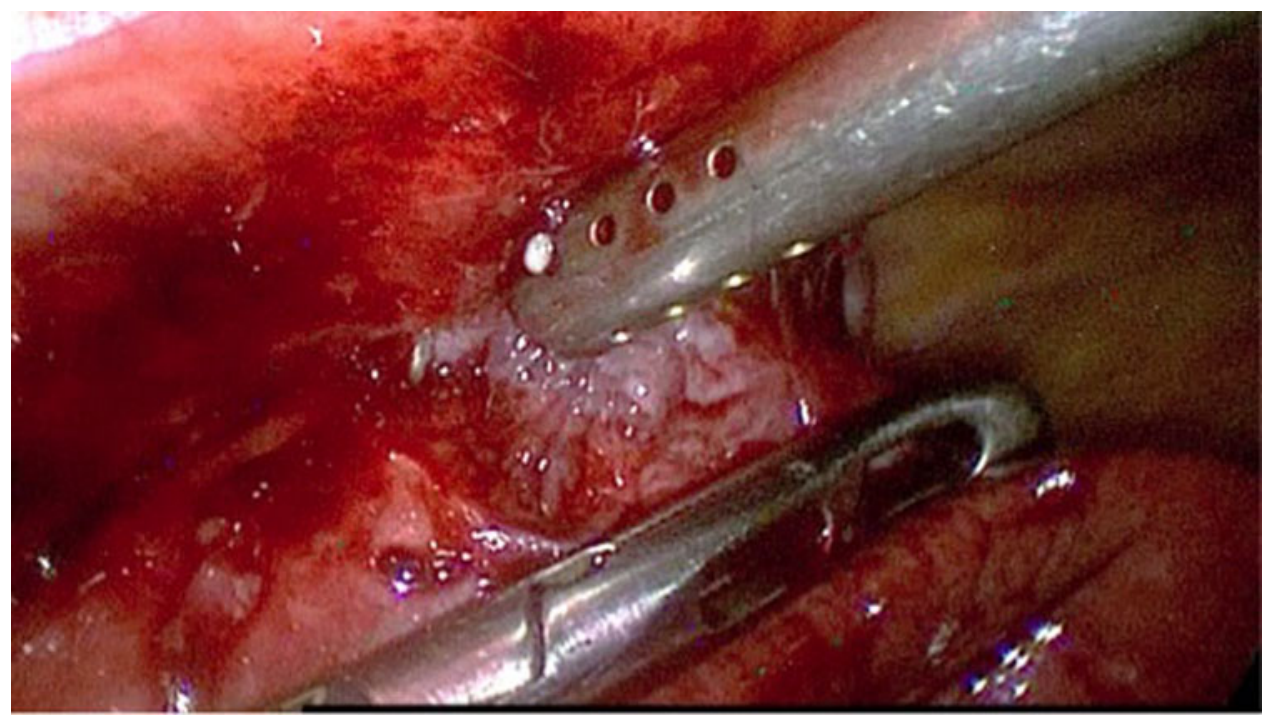

FIG. 2. Laparoscopic dissection of the fistulous tract. 


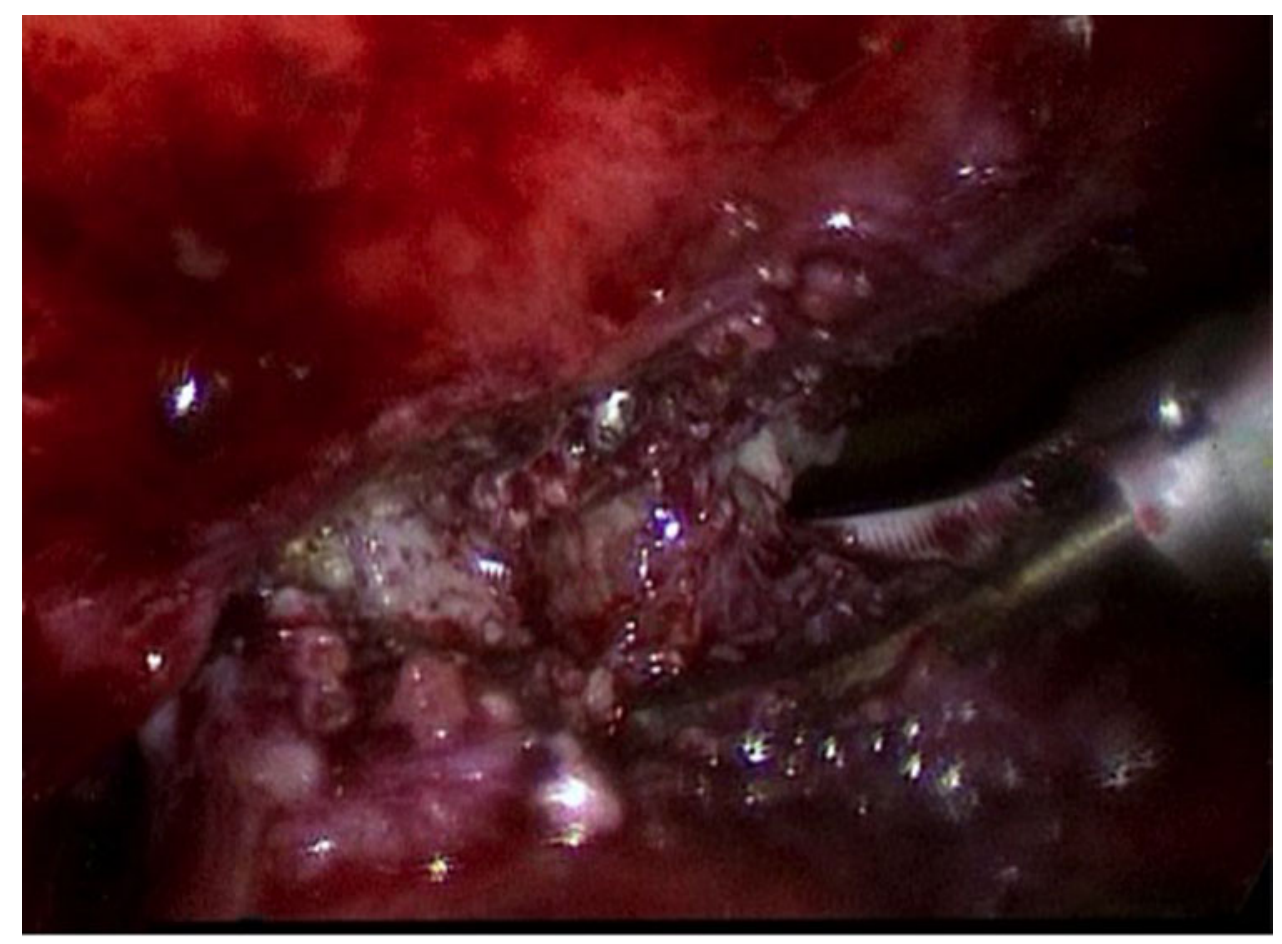

FIG. 3. The fistulous tract being excised laparoscopically.

this incidence increases in case of lost gallstones within the peritoneal cavity [1]. Reported complications ranged from pain and sinus formation to intra-abdominal abscess and intestinal fistulae [1-6]. Risk factors could play a major role in the complication process. Advanced age, pigmented stones, and number, location, and size of the stones are believed to increase the morbidity rate [5]. According to experimental animal studies, if bile leakage is associated with spilled stones, the tendency toward intra-abdominal abscess and adhesion formation is higher [2]. In one prospective study of 1,528 laparoscopic cholecystectomies over a 10-y period, the incidence of spilled gallstones was $3.8 \%$. Only $12 \%$ developed complications on long-term follow-up. These were treated either conservatively or by exploratory laparotomy. In this study, Tumer et al. [3] emphasized the necessity of informing the patient about the possibility of delayed complications. They have also recommended the need of conversion to laparotomy in cases of lost gallstones [3]. This was also recommended by Rice et al. [7] in 1997. Because serious complications could occur, such as colonic fistula as also reported in 1997 by Patterson et al. [6], immediate conversion should be considered as an option [6]. To the best of our knowledge, none of the reported cases in the English medical literature discussed re-laparoscopy in cases of delayed complication. In some cases, malignancy was suspected [4]. This could have a negative impact on patients, who can live in fear of having a serious life-threatening illness.

\section{Conclusion}

Perforated gallbladder is a common complication during laparoscopic cholecystectomy. Although morbidity is rare, serious complications can occur. Patients may suffer long-term complications, and some have had laparotomy recommended for symptom relief. The best treatment modality could not be demonstrated based on the few recent articles. Our case is unique, because a small quantity of bile was leaking through the trocar site, however, CT scan showed no evidence of communication with intra-hepatic ducts. Also a Glisson capsule rupture was not considered because the patient was stable and presented few months after the cholecystectomy. She was only bothered by the fistula. We recommended re-laparoscopy exploration, combined with fistula tract excision. Twelve months elapsed until the writing of this article and patient remains symptom-free.

\section{Author Disclosure Statement}

No competing financial interests exist.

\section{References}

1. Woodfield JC, Rodgers M, Windsor JA. Peritoneal gallstones following laparoscopic cholecystectomy Incidence, complications, and management. Surg Endosc 2004;18: 1200-1207.

2. Loffeld RJLF. The consequences of lost gallstones during laparoscopic cholecystectomy. Neth J Med 2006;64:10.

3. Tumer AR, Yüksek YN, Yasti AC, et al. Dropped gallstones during laparoscopic cholecystectomy: The consequences. World J Surg 2004:29:437-440.

4. Carlin CB, Kent RB, Laws HL. Spilled gallstonesComplications of abdominal-wall abscesses. Surg Endosc 1995;9:341-343.

5. Brockmann JG, Kocher T, Senninger NJ, Schurmann GM. Complications due to gallstones lost during laparoscopic 
Address correspondence to:

cholecystectomy. An analysis of incidence, clinical course, and management. Surg Endosc 2002;16:1226-1232.

6. Patterson EJ, Alexander G. Nagy AG. Don't cry over spilled gallstones? Complications of gallstones spilled during laparoscopic cholecystectomy: Case report and literature review. Can J Surg 1997;40:300-304.

7. Rice DC, Memon MA, Jamison RL, et al. Long-term consequences of intraoperative spillage of bile and gallstones during laparoscopic cholecystectomy. J Gastrointest Surg 1997;1:85-91.
Dr. Haydar A. Nasser

Department of General Surgery

Lebanese University

Beirut 961

Lebanon

E-mail: Derdyail@hotmail.com

\section{Abbreviations Used}

$\mathrm{CT}=$ computed tomography

Cite this article as: Hajj A, Nasser HA, Joudi I, Chalhoub D, Tayar C (2016) Glissonian capsule fistula after laparoscopic cholecystectomy. Surgical Infections Case Reports 1:1, 59-62, DOI: 10.1089/crsi.2016.0011 